\title{
Surgical palliative care disparities
}

\author{
Julian T. Rowe ${ }^{1}$, Fabian M. Johnston ${ }^{2}$ \\ ${ }^{1}$ School of Medicine, Johns Hopkins University, Baltimore, MD, USA; ${ }^{2}$ Division of Surgical Oncology, Department of Surgery, The Johns Hopkins \\ Hospital, Baltimore, MD, USA \\ Contributions: (I) Conception and design: Both authors; (II) Administrative support: FM Johnston; (III) Provision of study materials or patients: \\ Both authors; (IV) Collection and assembly of data: Both authors; (V) Data analysis and interpretation: Both authors; (VI) Manuscript writing: Both \\ authors; (VII) Final approval of manuscript: Both authors. \\ Correspondence to: Fabian M. Johnston, MD. 600 N. Wolfe Street, Baltimore, MD 21287, USA. Email: fjohnst4@jhmi.edu.
}

\begin{abstract}
Surgical palliative care is an interdisciplinary treatment modality that aims to decrease suffering and improve the quality of life of seriously ill surgical patients. Although surgical palliative care is increasingly being found to positively impact patient quality of life, disparities in surgical palliative care remain poorly defined. While the field of medical palliative care has demonstrated certain racial/ethnic, socioeconomic, and geographic groups are at higher risks for receiving worse palliative care, there is no analogous consensus in the field of surgical palliative care. This is largely secondary to a paucity of research focus in this field. Given that the aforementioned disparities experienced by minoritized patient populations has led to significant morbidity, it is important to understand and call attention to disparities existing within the field of surgical palliative care as well. To advance the knowledge of current healthcare shortcomings and progress towards equitable surgical palliative care, this paper reviews the current state of surgical palliative care disparities evidence, details gaps in knowledge, and highlights priorities for future surgical palliative care research. The articles identified in this review noted disparate surgical palliative care access and outcomes across various racial/ethnic groups, age ranges, socioeconomic classes, hospital populations, and regions. However, evidence scarcity necessitates more robust research be performed to adequately identify at risk groups and understand the factors supporting disparity development.
\end{abstract}

Keywords: Healthcare disparities; surgery; palliative

Submitted Dec 02, 2020. Accepted for publication Aug 03, 2021.

doi: 10.21037/apm-20-2394

View this article at: https://dx.doi.org/10.21037/apm-20-2394

\section{Introduction}

Healthcare disparities for certain priority population groups, such as racial/ethnic minorities and rural populations, have been widely documented in both medical and surgical fields (1-5). Worse access, quality of care, and outcomes all contribute to a significantly increased health burden in these groups $(6,7)$. Unsurprisingly, previous studies have also established that minority groups experience worse care in palliative care domains such as symptom management, quality of life (QOL) improvement, advanced care planning, and alignment of doctor and patient healthcare goals $(8,9)$. Unfortunately, less is known about disparities in the subset of palliative care dealing with surgical patients experiencing a significantly decreased QOL, commonly referred to as surgical palliative care. Disparities in surgical palliative care specifically may be clinically magnified by the fact that surgical patients often carry higher burdens of disease, shortened survival, and a significantly decreased QOL compared to their medical counterparts (10). To emphasize the importance of the issue, the NIH, and American College of Surgeons in 2016 designated improving patient centeredness and palliative care as one of the top 5 surgical disparities research priorities (11).

Currently, surgical palliative care is an understudied field with a patient base experiencing significant illness. To promote understanding of what disparities exist, where they exist, and how they might impact different populations, this 
paper aimed to review and summarize the current literature on disparities in surgical palliative care.

\section{Definitions}

To understand the scope of the surgical palliative care field, it is important to highlight a few key definitions. Surgical palliative care is an interdisciplinary treatment modality that aims to improve the QOL of patients suffering from serious surgical illness-i.e., those who are likely to die from their illness or be significantly hindered by it $(10,12)$. Importantly, surgical palliative care is an approach to care, not just the act of surgery itself. Palliative surgery is just one tool in the surgical palliative care toolkit, and therapies besides surgery, such as emotional support and pain management, can be used to improve the QOL for surgical patients and their families. An 11-item criterion was curated and validated by Lee $e t a l$. to define specific serious illness conditions and included ailments such as end-stage renal disease and an ASA Risk Score class of IV or V (10). In short, surgical patients with serious illness are the unique population of study, and the changing needs of this population based on different factors, such as race, age, or geography, are important to understand.

\section{Exploring access disparities in fields related to surgical palliative care}

Before exploring the disparities specifically pertaining to surgical palliative care, it is of use to highlight the widely established disparities in the field of palliative care as a whole. Multiple disparities at different levels of healthcare play a role in decreasing access to palliative care. These disparities have been identified at individual patientphysician and hospital/systemic levels.

At the individual patient-physician level, race, age, physician biases including reluctance to refer, and communication barriers have all been shown to significantly decrease palliative care access. With regards to racial differences, Black and Hispanic patients have been shown to receive lower rates of palliative care compared to White patients (13-15). Another study found that Black patients tend to be sicker before receiving palliative care, and are less likely to leave from a hospital alive after having received palliative care (16). Factors that play a role in generating this racial disparity include differences in utilization and social factors. More specifically treatment preferences, cultural preferences for end of life care, and knowledge differences have been suggested to decrease racial utilization of hospice care services (17). Other social determinants that generate disparities in palliative care include financial/ insurance barriers and misconceptions/lack of information about palliative care (18). Taken together, this supports the notion that race plays a complex, multidimensional role in the reception of palliative healthcare.

Several communication barriers, mediated by patient or physician preferences or linguistic differences, have also been shown to significantly influence palliative care access. From the patient perspective, patients reported a desired to focus on life-preserving options rather than death-related domains as well as a lack of understanding of preferred care for serious illness as reasons for avoiding initiating palliative care conversations (19). When patients or physicians do initiate palliative care conversations, barriers in language interpretations can result in patient misunderstanding of their disease process along with worse QOL if patients' goals of care do not align with their physicians' actions $(20,21)$. For other patients, difficulty understanding medical terminology or palliative care options decrease access. Within the senior patient population specifically, it has been shown that inability to accurately hear health-related conversations can lead to decreased comprehension (22). Taken together these factors produce a system in which palliative care might not be prioritized.

At the hospital level, there is evidence that disparities persist outside of factors directly attributable to one patient or physician. When Faigle et al. explored system-level factors contributing to racial disparities in palliative care use after stroke, it was found that White and Black patients at predominantly minority-serving hospitals were less likely to receive palliative care compared to White and Black patients at predominantly non-minority serving hospitals (23). Subsequent studies have continued to highlight that patients at predominantly minority-serving hospitals are less likely to receive palliative care, regardless of race (24).

Finally, geographic limitations play a significant systemic role in limiting palliative care access. Ideally palliative care is administered as an interdisciplinary approach that addresses patient and family physical, psychosocial, and spiritual concerns. Rural medicine in particular commonly falls short of this gold standard because of inadequate primary care physician education and training, inadequate preparation for symptom control and emotional counseling, and primary care physician difficulty in accessing specialist services when they are needed $(25,26)$. Geographic distance has also been noted as creating time constraints 
that decreases administration of rural palliative care and decreases the professional development required to learn and maintain an understanding of the use of palliative care $(25,26)$. Notably, the 2019 State-By-State Report Card on Access to Palliative Care in Our Nation's Hospitals reported that $90 \%$ of hospitals with palliative care are in urban areas. Further, only $17 \%$ of rural hospitals with 50 or more beds reported having palliative care programs. In short, where you live matters in terms of access to palliative care (27).

Although palliative care in medical and surgical populations is related in mission, it would be shortsighted to immediately begin investing resources to increase surgical palliative care access without acknowledging the specific barriers to access in surgical populations. While we can learn from the disparities experienced above, it is important to note how the two populations differ significantly. Surgical patients not only suffer from serious illness in need of direct intervention, they also deal with the added pressures of understanding and making quick care decisions during an accelerated perioperative time frame (28). For older adults, treatment decisions typically become more complex with advancing age. Patients must decide if they prefer aggressive treatments that negatively impact their QOL in the short term for a potential increase in lifespan versus QOL-conserving treatments without expected survival benefit (29). These considerations are especially difficult and sensitive to personal patient values due to the scarcity of data comparing long term QOL and function maintenance across operative and nonoperative treatments (28). The shortened time frame, added considerations that come with perioperative advance care planning, and causal disease differences necessitate understanding potential disparities in care from a surgical palliative care lens specifically.

\section{Exploring the disparities in surgical palliative care access}

Barriers to accessing care are integral drivers of worse quality of care overall and specific disparities. Mediators to health care access can range from individual physician decisions, to cultural preferences, to structural factors. Characterizing known disparities in surgical palliative care access among different populations is essential to providing quality healthcare for all.

\section{Evidence for disparities in surgical palliative care access}

Multiple studies have consistently shown that across all populations, surgical patients are less likely to receive palliative care compared to medical patients (30-32). Lack of education, physician biases, and a "fix it" culture have been described as fundamental reasons for this discrepancy. One reason for the reluctance to refer is a lack of education. Surgeons report a lack of training to engage in palliative discussions (33). With regards to biases, some physicians' views that palliative care is most useful at the end of life, coupled with views that early use of palliative care is a sign of giving up contributes to the variable and typically late usage of palliative care (34-38). There is a strong culture in surgery to fix an established problem and to save lives. This has led to palliative care being seen as both secondary to and a failure of the "fix it" primary mission (39). Mosenthal et al. noted that external factors, such as the use of surgical morbidity and mortality as healthcare quality indicators and the public reporting of this data further reinforce the sense of personal failure surgeons feel when poor outcomes are experienced (39). Such external factors further delay prioritization of palliative goals of card that don't aim treat a patient's condition rather than improve their QOL (39).

Novel research has begun to highlight specific surgical patient populations at risk for decreased palliative care access, defining "priority populations" for surgical palliative care. A review of the national inpatient sample by Heller et al. found that predictors for surgical palliative care administration are partially similar to those described for the palliative care field as a whole (40). Patients at a higher risk for death such as increased age and comorbidities, receiving care in an academic not for profit, large hospital, and living in a region outside of the northeast all predicted an increased likelihood of receiving surgical palliative care (40). It is notable that investigators found the northeast a negative predictor of surgical palliative care reception since the center to advance palliative reports patients in the northeast have access to significantly more hospital palliative care programs (27). The researchers suggested geographic differences and biases in goals of care, decisions making, and expectations of surgical outcomes among surgeons or patients were potential reasons for this disparity.

Unfortunately, few studies have looked at racial, ethnic, and socioeconomic disparities in palliative care delivery to surgical populations specifically. Although surgical palliative care access disparities have been described, there is still much to learn about who has access to palliative care and how it is different among different populations. For racial/ ethnic minorities, there is currently no consensus on the influence race plays on surgical palliative care access. One 
study determined that African American and Hispanic patients are less likely to receive palliative interventions while waiting for liver transplantation (41). Regarding socioeconomic status, another study found that patients with lower SES were less likely to be referred for palliative colorectal stenting for malignant obstructions (42).

\section{Evidence gaps in understanding surgical palliative care access disparities}

The major gaps of knowledge regarding disparities in surgical palliative care access surround effective communication and decision making techniques and efficient palliative healthcare delivery (28). With regards to communication, an expert consensus conference hosted by the National Institutes of Health and National Palliative Care Research Center highlighted the need for better communication techniques to help surgeons guide advanced care planning conversations, complete conversations detailing patient's desired outcomes from palliative procedures, and provide reasonable prognostic predictions in times of uncertainty (28). Without the knowledge of how to guide these conversations, surgeons must rely on assumptions of patient preferences and feelings of what seems right to disclose.

With regards to palliative healthcare delivery, the group noted that efficient scalable models and screening tools must be made to allow for the seamless integration of palliative care into surgical care (28). Whether or not better delivery of palliative care will impact barriers to palliative care access such as patient knowledge of resources, resource limitation, of palliative care value misunderstandings has yet to be determined. Future research and solutions should be designed in such a way as to decrease the wholistic individual, hospital level, and systemic barriers while concurrently prioritizing disparities studies research.

\section{What's next in surgical palliative care access}

Moving forward, the field of surgical palliative care must expand its access to all patients while also investing in specific disparity identification, research, and solution discovery, the three domains of disparities research (43). The relationship is mutually beneficial as increased access will drive any disparity findings and disparity findings will inform equitable surgical palliative care research design. Previous research has already defined potentially effective tools to increase palliative care access at an individual and hospital level, including the palliative care triangle method and palliative care triggers. The palliative care triangle is a decision making model that includes surgeons, patients, and their families in conversations to help guide individual decisions regarding palliative care (44). The model helps care teams and patients make decisions by taking patients' "complaints, values, and emotional support" into account versus surgical and medical treatment options (44). By helping to select patients with the most to gain from palliative operations, the method is cited as a means to help overcome the lack of formal training surgeons have in palliative care in a cost efficient and consistent manner $(44,45)$. As a second solution to increase access, multiple studies have successfully implemented a system of triggers that clearly characterize opportunities to iterate palliative care into a surgical patient's healthcare (46-48). In addition to increasing the application of the palliative triangle and triggers, surgeons should be supported further with education regarding the utility of palliative care, how to provide primary palliative care, and the necessity to treat patients individually to help avoid stereotyping palliative care as a "last option". Lastly, future studies must also determine potential systemic level access barriers solutions such as investing money to increase palliative care programs within states and regions with few palliative care containing hospitals. As access to palliative medicine is increased, it must be kept in mind that these barriers could affect priority populations differentially. It will become increasingly important to sub stratify research by commonly marginalized priority populations to determine if these increases in access are being shared equally.

\section{Disparities in surgical palliative care outcomes}

Studies have shown early integration of surgical palliative care into disease management improves symptom management, advanced care planning, and coordination of care while reducing hospital length of stay, odds of readmissions, and healthcare cost (49-54). Although it is not currently possible to determine which surgical palliative interventions and outcomes are most effective due to study heterogeneity, it remains clear that surgical palliative care has a positive impact (30). As palliative interventions and outcomes are standardized, it is important to also detail any potential disparities in surgical palliative care outcomes among priority populations.

\section{Evidence for disparities in surgical palliative care outcomes}

Although there is no current consensus on whether certain 


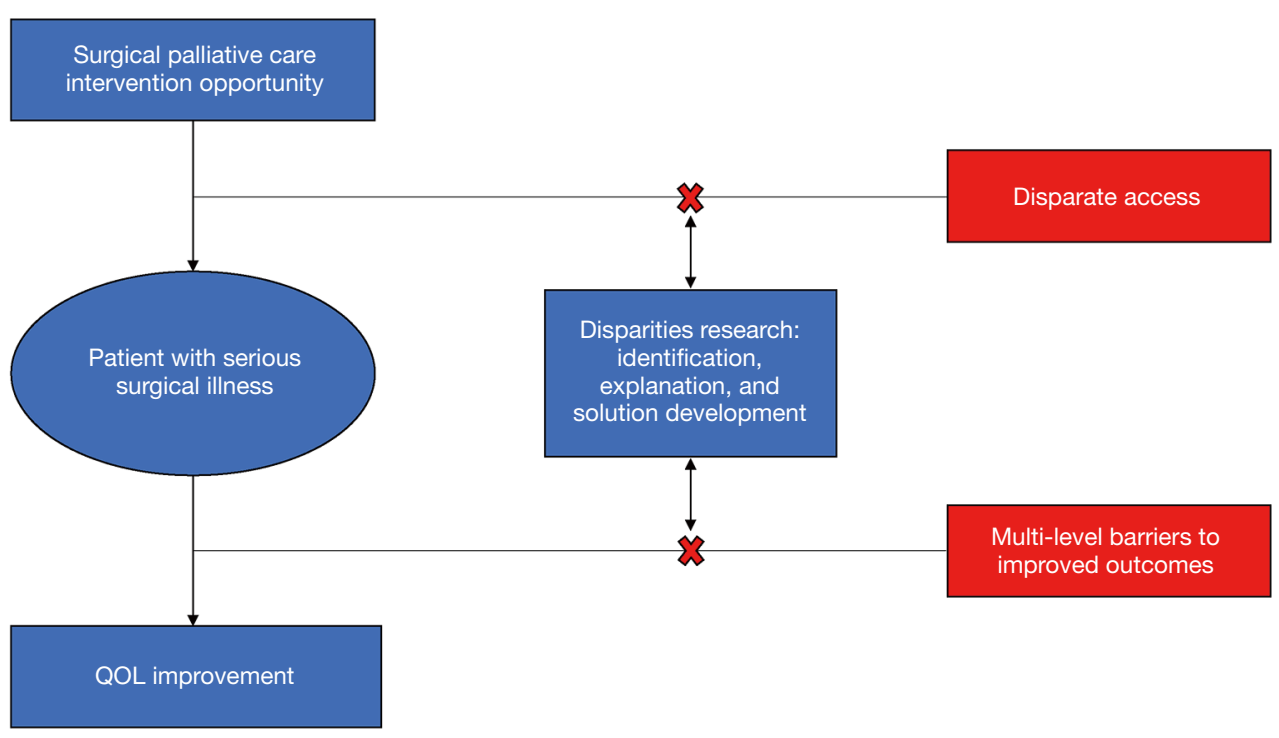

Figure 1 Targets for disparities research in surgical palliative care. QOL, quality of life.

priority populations experience worse surgical palliative care outcomes compared to non-priority populations, some research suggests differences exist at systemic and individual levels. At a systemic level, Hammad et al. found that treatment at a non-academic center was a negative predictor of survival when comparing palliative interventions on patients with hepatocellular carcinoma (55). At an individual level, the study found that advanced age and multiple comorbidities were negative predictors of survival following palliative intervention (55). Across racial/ ethnic priority populations, studies have shown worse pain, communication, satisfaction, and advance care outcomes in the African American community, including surgical patient populations (56). Another study highlighting racial/ disparities found that only $40.4 \%$ of Black families rated their lost ones' end of life care after high-risk surgery as excellent compared to $54 \%$ of White families (57). This theme that African American families are less satisfied with their care compared to White families has been well described in the literature $(58,59)$. Although these emerging findings begin to point to populations with disparate surgical palliative care outcomes, it is important to appreciate the limitations of surgical palliative care research. For example, when comparing families' approval of their loved one's end of life care, it can be difficult to pinpoint the most important driving factors for the differential dissatisfaction. More specifically, it is not clear if differential treatment and a better experience was received during the last few weeks of care or if palliative care was different throughout the patient's disease course. Further, patient-level differences in palliative care expectations or awareness about treatment options could drive differential ratings. There are a multitude of nuances driving surgical palliative care outcomes and disparities research must be careful to account for these nuances. By appreciating these components driving disparities we can move past identification of potential underlying causes, and more efficiently detail solutions.

\section{Evidence gaps and future directions for understanding surgical palliative care outcomes disparities}

Much work still needs to be done to determine if significant disparities in surgical palliative care outcomes exist across different populations. Most of the current surgical palliative care research invests in understanding its proper indications, innovating new ways to increase accessibility, and defining what outcomes matter $(30,60,61)$. Because of the extensive healthcare disparities experienced throughout other fields of medicine, it is also important to recognize how surgical palliative care advances might disparately affect various at-risk groups such as rural populations, racial/ethnic minorities, and older adults. Future actions should prioritize defining generalizable outcomes, performing multiinstitutional high-quality studies, and investing in disparity research. The domains to invest in to move forward towards equitable surgical palliative care are summarized in Figure 1. 
From 1994 to 2014, there were only 25 studies centered on palliative care interventions for surgical patients (30). This clearly denotes the need for more surgical palliative care research and highlight great opportunity for growth including opportunities to address disparities.

\section{Conclusions}

Emerging evidence in palliative care research suggests that disparate palliative care access and outcomes exist for certain surgical patient populations on the basis of race, age, socioeconomic status, and geographical region. However, more work needs to be done to better define these differences in surgical patient populations. Although disparities data is trending towards similar atrisk populations as seen in medical palliative care, it is not enough to extrapolate findings from the medical field to design solutions for surgical palliative care disparities. Future research must advance accessibility and outcomes measurement tools specific to surgical palliative care while considering the different populations that are affected within each study. As future research establishes more robust data, disparities can be clearly identified, understood, and addressed effectively. Future surgical palliative care disparity studies have a valuable task ahead of them with the potential to shape policy and clinical practice that aims to provide equitable QOL improving care to uniquely vulnerable health care populations.

\section{Acknowledgments}

The authors wish to thank Jaime Blanck and Rachael Lebo for their insightful article search guidance.

Funding: The project described was supported by grant number 1K08HS024736-01 from the Department of Health \& Human Services Agency for Healthcare Research and Quality.

\section{Footnote}

Provenance and Peer Review: This article was commissioned by the by the editorial office, Annals of Palliative Medicine for the series "Palliative Care and Surgery". The article has undergone external peer review.

Conflicts of Interest: The authors have completed the ICMJE uniform disclosure form (available at https://apm. amegroups.com/article/view/10.21037/apm-20-2394/coif).
The series "Palliative Care and Surgery" was commissioned by the editorial office without any funding or sponsorship. FMJ served as the unpaid Guest Editor of the series, and served as an unpaid editorial board member of Annals of Palliative Medicine from Oct 2019 to Sep 2021. The authors have no other conflicts of interest to declare.

Ethical Statement: The authors are accountable for all aspects of the work in ensuring that questions related to the accuracy or integrity of any part of the work are appropriately investigated and resolved.

Open Access Statement: This is an Open Access article distributed in accordance with the Creative Commons Attribution-NonCommercial-NoDerivs 4.0 International License (CC BY-NC-ND 4.0), which permits the noncommercial replication and distribution of the article with the strict proviso that no changes or edits are made and the original work is properly cited (including links to both the formal publication through the relevant DOI and the license). See: https://creativecommons.org/licenses/by-nc-nd/4.0/.

\section{References}

1. Douthit N, Kiv S, Dwolatzky T, et al. Exposing some important barriers to health care access in the rural USA. Public Health 2015;129:611-20.

2. FitzGerald C, Hurst S. Implicit bias in healthcare professionals: a systematic review. BMC Med Ethics 2017;18:19.

3. Wheeler SM, Bryant AS. Racial and Ethnic Disparities in Health and Health Care. Obstet Gynecol Clin North Am 2017;44:1-11.

4. Wood MH, Carlin AM, Ghaferi AA, et al. Association of Race With Bariatric Surgery Outcomes. JAMA Surg 2019;154:e190029.

5. Benavidez OJ, Gauvreau K, Jenkins KJ. Racial and ethnic disparities in mortality following congenital heart surgery. Pediatr Cardiol 2006;27:321-8.

6. DiBardino DJ, Pasquali SK, Hirsch JC, et al. Effect of sex and race on outcome in patients undergoing congenital heart surgery: an analysis of the society of thoracic surgeons congenital heart surgery database. Ann Thorac Surg 2012;94:2054-9; discussion 2059-60.

7. Haider AH, Scott VK, Rehman KA, et al. Racial disparities in surgical care and outcomes in the United States: a comprehensive review of patient, provider, and systemic factors. J Am Coll Surg 2013;216:482-92.e12. 
8. Smith AK, McCarthy EP, Paulk E, et al. Racial and ethnic differences in advance care planning among patients with cancer: impact of terminal illness acknowledgment, religiousness, and treatment preferences. J Clin Oncol 2008;26:4131-7.

9. Anderson KO, Green CR, Payne R. Racial and ethnic disparities in pain: causes and consequences of unequal care. J Pain 2009;10:1187-204.

10. Lee KC, Walling AM, Senglaub SS, et al. Defining Serious Illness Among Adult Surgical Patients. J Pain Symptom Manage 2019;58:844-50.e2.

11. Haider AH, Dankwa-Mullan I, Maragh-Bass AC, et al. Setting a National Agenda for Surgical Disparities Research: Recommendations From the National Institutes of Health and American College of Surgeons Summit. JAMA Surg 2016;151:554-63.

12. Dunn GP. Surgical Palliative Care, Surgical Palliative Care. In: Current Surgical Therapy 13th ed. Philadelphia, PA: Elsevier, 2020:1365-70.

13. Murthy SB, Moradiya Y, Hanley DF, et al. Palliative Care Utilization in Nontraumatic Intracerebral Hemorrhage in the United States. Crit Care Med 2016;44:575-82.

14. Wen Y, Jiang C, Koncicki HM, et al. Trends and Racial Disparities of Palliative Care Use among Hospitalized Patients with ESKD on Dialysis. J Am Soc Nephrol 2019;30:1687-96.

15. Rising ML, Hassouneh DS, Lutz KF, et al. Hispanic Hospice Utilization: Integrative Review and Meta-analysis. J Health Care Poor Underserved 2019;30:468-94.

16. Lee K, Gani F, Canner JK, et al. Racial Disparities in Utilization of Palliative Care Among Patients Admitted With Advanced Solid Organ Malignancies. Am J Hosp Palliat Care 2021;38:539-46.

17. Johnson KS. Racial and ethnic disparities in palliative care. J Palliat Med 2013;16:1329-34.

18. Gardner DS, Doherty M, Bates G, et al. Racial and Ethnic Disparities in Palliative Care: A Systematic Scoping Review. Fam Soc 2018;99:301-16.

19. Curtis JR, Engelberg RA, Wenrich MD, et al. Communication about palliative care for patients with chronic obstructive pulmonary disease. J Palliat Care 2005;21:157-64.

20. Kim G, Worley CB, Allen RS, et al. Vulnerability of older Latino and Asian immigrants with limited English proficiency. J Am Geriatr Soc 2011;59:1246-52.

21. Wagner GJ, Riopelle D, Steckart J, et al. Provider communication and patient understanding of life-limiting illness and their relationship to patient communication of treatment preferences. J Pain Symptom Manage 2010;39:527-34.

22. Periyakoil VS, Neri E, Kraemer H. Patient-Reported Barriers to High-Quality, End-of-Life Care: A Multiethnic, Multilingual, Mixed-Methods Study. J Palliat Med 2016;19:373-9.

23. Faigle R, Ziai WC, Urrutia VC, et al. Racial Differences in Palliative Care Use After Stroke in Majority-White, Minority-Serving, and Racially Integrated U.S. Hospitals. Crit Care Med 2017;45:2046-54.

24. Cole AP, Nguyen DD, Meirkhanov A, et al. Association of Care at Minority-Serving vs Non-Minority-Serving Hospitals With Use of Palliative Care Among Racial/ Ethnic Minorities With Metastatic Cancer in the United States. JAMA Netw Open 2019;2:e187633.

25. Robinson CA, Pesut B, Bottorff JL, et al. Rural Palliative Care: A Comprehensive Review. J Palliat Med 2009. [Epub ahead of print]. doi: 10.1089/jpm.2008.0228.

26. Evans R, Stone D, Elwyn G. Organizing palliative care for rural populations: a systematic review of the evidence. Fam Pract 2003;20:304-10.

27. Morrison RS, Meier DE, Rogers M, et al. America's Care of Serious Illness: 2019 State-by-State Report Card on Access to Palliative Care in Our Nation's Hospitals. New York: Center to Advance Palliative Care 2019.

28. Lilley EJ, Cooper Z, Schwarze ML, et al. Palliative Care in Surgery: Defining the Research Priorities. J Palliat Med 2017;20:702-9.

29. Berian JR, Rosenthal RA, Baker TL, et al. Hospital Standards to Promote Optimal Surgical Care of the Older Adult: A Report From the Coalition for Quality in Geriatric Surgery. Ann Surg 2018;267:280-90.

30. Lilley EJ, Khan KT, Johnston FM, et al. Palliative Care Interventions for Surgical Patients: A Systematic Review. JAMA Surg 2016;151:172-83.

31. Olmsted CL, Johnson AM, Kaboli P, et al. Use of palliative care and hospice among surgical and medical specialties in the Veterans Health Administration. JAMA Surg 2014;149:1169-75.

32. Gani F, Enumah ZO, Conca-Cheng AM, et al. Palliative Care Utilization among Patients Admitted for Gastrointestinal and Thoracic Cancers. J Palliat Med 2018;21:428-37.

33. Suwanabol PA, Reichstein AC, Suzer-Gurtekin ZT, et al. Surgeons' Perceived Barriers to Palliative and End-ofLife Care: A Mixed Methods Study of a Surgical Society. J Palliat Med 2018;21:780-8.

34. Hawley P. Barriers to Access to Palliative Care. Palliat 
Care 2017;10:1178224216688887.

35. Morell E, Thompson J, Rajagopal S, et al. Congenital Cardiothoracic Surgeons and Palliative Care: A National Survey Study. J Palliat Care 2021;36:17-21.

36. Blumenthal B, Lee CW, Vitous CA, et al. Barriers to palliative care use among surgical patients: perspectives of practicing surgeons across Michigan. Ann Palliat Med 2021;10:1122-32.

37. Galante JM, Bowles TL, Khatri VP, et al. Experience and attitudes of surgeons toward palliation in cancer. Arch Surg 2005;140:873-8; discussion 878-80.

38. Suwanabol PA, Kanters AE, Reichstein AC, et al. Characterizing the Role of U.S. Surgeons in the Provision of Palliative Care: A Systematic Review and MixedMethods Meta-Synthesis. J Pain Symptom Manage 2018;55:1196-1215.e5.

39. Mosenthal AC, Weissman DE, Curtis JR, et al. Integrating palliative care in the surgical and trauma intensive care unit: a report from the Improving Palliative Care in the Intensive Care Unit (IPAL-ICU) Project Advisory Board and the Center to Advance Palliative Care. Crit Care Med 2012;40:1199-206.

40. Heller DR, Jean RA, Chiu AS, et al. Regional Differences in Palliative Care Utilization Among Geriatric Colorectal Cancer Patients Needing Emergent Surgery. J Gastrointest Surg 2019;23:153-62.

41. Nguyen GC, Segev DL, Thuluvath PJ. Racial disparities in the management of hospitalized patients with cirrhosis and complications of portal hypertension: a national study. Hepatology 2007;45:1282-9.

42. Okafor PN, Stobaugh DJ, Wong Kee Song LM, et al. Socioeconomic Inequalities in the Utilization of Colorectal Stents for the Treatment of Malignant Bowel Obstruction. Dig Dis Sci 2016;61:1669-76.

43. Kilbourne AM, Switzer G, Hyman K, et al. Advancing health disparities research within the health care system: a conceptual framework. Am J Public Health 2006;96:2113-21.

44. Miner TJ, Cohen J, Charpentier K, et al. The palliative triangle: improved patient selection and outcomes associated with palliative operations. Arch Surg 2011;146:517-22.

45. Sutton JE Jr. The palliative care triangle: a strategy to help make difficult surgical decisions. Arch Surg 2011;146:522-3.

46. Bradley C, Weaver J, Brasel K. Addressing access to palliative care services in the surgical intensive care unit. Surgery 2010;147:871-7.
47. Finkelstein M, Goldstein NE, Horton JR, et al. Developing triggers for the surgical intensive care unit for palliative care integration. J Crit Care 2016;35:7-11.

48. Chernock B, Hwang F, Berlin A, et al. Emergency abdominal surgery in patients presenting from skilled nursing facilities: Opportunities for palliative care. Am J Surg 2020;219:1076-82.

49. Ernst KF, Hall DE, Schmid KK, et al. Surgical palliative care consultations over time in relationship to systemwide frailty screening. JAMA Surg 2014;149:1121-6.

50. Mosenthal AC, Murphy PA, Barker LK, et al. Changing the culture around end-of-life care in the trauma intensive care unit. J Trauma 2008;64:1587-93.

51. Chatterjee K, Harrington S, Sexton K, et al. Impact of Palliative Care Utilization for Surgical Patients Receiving Prolonged Mechanical Ventilation: National Trends (20092013). Jt Comm J Qual Patient Saf 2020;46:493-500.

52. Wentlandt K, Weiss A, O'Connor E, et al. Palliative and end of life care in solid organ transplantation. Am J Transplant 2017;17:3008-19.

53. Baumann AJ, Wheeler DS, James M, et al. Benefit of Early Palliative Care Intervention in End-Stage Liver Disease Patients Awaiting Liver Transplantation. J Pain Symptom Manage 2015;50:882-6.e2.

54. Lilley EJ, Lee KC, Scott JW, et al. The impact of inpatient palliative care on end-of-life care among older trauma patients who die after hospital discharge. J Trauma Acute Care Surg 2018;85:992-8.

55. Hammad AY, Robbins JR, Turaga KK, et al. Palliative interventions for hepatocellular carcinoma patients: analysis of the National Cancer Database. Ann Palliat Med 2017;6:26-35.

56. Boucher NA, Raghavan M, Smith A, et al. Palliative Care in the African American Community \#204. J Palliat Med 2016;19:228-30.

57. Yefimova M, Aslakson RA, Yang L, et al. Palliative Care and End-of-Life Outcomes Following High-risk Surgery. JAMA Surg 2020;155:138-46.

58. Rhodes RL, Teno JM, Connor SR. African American bereaved family members' perceptions of the quality of hospice care: lessened disparities, but opportunities to improve remain. J Pain Symptom Manage 2007;34:472-9.

59. Welch LC, Teno JM, Mor V. End-of-life care in black and white: race matters for medical care of dying patients and their families. J Am Geriatr Soc 2005;53:1145-53.

60. Lee KC, Walling AM, Senglaub SS, et al. Improving Serious Illness Care for Surgical Patients: Quality Indicators for Surgical Palliative Care. Ann Surg 2020. doi: 
10.1097/SLA.0000000000003894. [Epub ahead of print]. 61. Søreide JA, Tholfsen T, Karlsen LN, et al. Palliative surgical outcome score (PSOS) in patients treated

Cite this article as: Rowe JT, Johnston FM. Surgical palliative care disparities. Ann Palliat Med 2022;11(2):862-870. doi: 10.21037/apm-20-2394 palliatively with self-expanding metal stent (SEMS) for malignant incurable colorectal obstruction. Surg Oncol 2019;29:134-9. 